\title{
Efficiently Computable Endomorphisms for Hyperelliptic Curves
}

\author{
David R. Kohel, Benjamin A. Smith \\ School of Mathematics and Statistics, \\ The University of Sydney \\ \{kohel, bens\}@maths . usyd.edu. au
}

\begin{abstract}
Elliptic curves have a well-known and explicit theory for the construction and application of endomorphisms, which can be applied to improve performance in scalar multiplication. Recent work has extended these techniques to hyperelliptic Jacobians, but one obstruction is the lack of explicit models of curves together with an efficiently computable endomorphism. In the case of hyperelliptic curves there are limited examples, most methods focusing on special CM curves or curves defined over a small field. In this article we describe three infinite families of curves which admit an efficiently computable endomorphism, and give algorithms for their efficient application.
\end{abstract}

Keywords. Hyperelliptic curve cryptography, efficiently computable endomorphisms.

\section{Introduction}

The use of efficiently computable endomorphisms for speeding up point multiplication on elliptic curves is well-established for elliptic curves and more recently has been used for hyperelliptic curves. Koblitz [10] proposed $\tau$-adic expansions of the Frobenius endomorphism on curves over a small finite fields. Gallant, Lambert, and Vanstone [6] later proposed using an expression

$$
[k] P=\left[k_{0}\right] P+\left[k_{1}\right] \phi(P)
$$

on more general curves to evaluate multiplication by $k$ on a point $P$, using an efficiently computable endomorphism $\phi$. Various improvements and combinations of these methods have been proposed for both elliptic and hyperelliptic curves 11172 .

One feature of elliptic curves, not available for multiplicative groups of finite fields, is the freedom to choose a parameter: geometrically they form a onedimensional family, parametrized by the $j$-invariant. Restriction to curves of a special form destroys this degree of freedom. While no proof exists that special curves, CM curves or Koblitz curves are less insecure, these nonrandom curves can be qualitatively distinguished from their nonrandom cousins in terms of their endomorphism rings. Thus preference is often given to curves randomly selected over a large finite field when performance is not the determining issue. 
In contrast, hyperelliptic curves of genus $g$ admit a much larger degree of freedom. In genus 2 , they form a three dimensional family: curves with different classifying triple of invariants $\left(j_{1}, j_{2}, j_{3}\right)$ can not be isomorphic over any extension field. Until the recent work of Takashima 19, the only curves proposed for cryptographic use with efficiently computable endomorphisms are either the CM curves with exceptional automorphisms - the analogues of elliptic curves $y^{2}=$ $x^{3}+a$ or $y^{2}=x^{3}+a x$ - or Koblitz curves - curves defined over a small field with point on the Jacobian taken over a large prime degree extension (see Park et al. [15] for the former and Lange [11] for the latter). Besides the notable exceptions of CM curves with exceptional automorphisms, curves with CM have been exploited for point counting but not for their endomorphism ring structure, for lack of a constructive theory of efficiently computable endomorphisms.

In this work, we address the problem of effective algorithms for endomorphisms available on special families of curves. We describe three families, of dimensions 1, 1, and 2 respectively, of curves whose Jacobians admit certain real endomorphisms. First, we introduce the general framework for constructing endomorphisms via correspondences derived from covering curves. Subsequently, we provide a one-dimensional family derived from Artin-Schreier covers, then describe a construction of Tautz, Top, and Verberkmoes 20 for a one-dimensional family of curves with explicit endomorphisms deriving from cyclotomic covers. Finally, we describe an elegant construction of Mestre [14 from which we obtain a two-dimensional family of curves whose Jacobians admit explicit endomorphisms, derived from covers of elliptic curves. In each case we develop explicit algorithms for efficient application of the endomorphism, suitable for use in a GLV decomposition. Independently, Takashima [19 provided an efficient algorithm for endomorphisms in the latter family (in terms of variants of Brumer and Hashimoto) with real multiplication by $(1+\sqrt{5}) / 2$. These families provide a means of generating curves randomly selected within a large family, yet which admit efficiently computable endomorphisms.

\section{Arithmetic on Hyperelliptic Jacobians}

In the sequel we denote by $X / k$ a hyperelliptic curve of genus $g_{X}$ in the form

$$
v^{2}=f(u)=u^{2 g_{X}+1}+c_{2 g_{X}} u^{2 g_{X}}+\cdots+c_{0},
$$

with each $c_{i}$ in $k$, which we require to be a field of characteristic not 2 . The Jacobian of $X$, denoted $\operatorname{Jac}(X)$, is a $g_{X}$-dimensional variety whose points form an abelian group. Let $\mathcal{O}$ denote the point at infinity of $X$. Each point $P$ on $\operatorname{Jac}(X)$ may be represented by a divisor on $X$, that is, as a formal sum of points

$$
P=\sum_{i=1}^{m}\left[P_{i}\right]-m[\mathcal{O}]=\sum_{i=1}^{m}\left[\left(u_{i}, v_{i}\right)\right]-m[\mathcal{O}],
$$

where $m \leq g_{X}$. We say such a divisor is semi-reduced if $\left(u_{i}, v_{i}\right) \neq\left(u_{j},-v_{j}\right)$ for all $i \neq j$. For a point to be defined in $\operatorname{Jac}(X)(k)$, its divisor must be Galois-stable; 
the representation as a divisor has the disadvantage that the individual points $\left(u_{i}, v_{i}\right)$ may be defined only over some finite extension $K / k$. Thus, for computations, we use instead the Mumford representation for divisors, identifying $P$ with the ideal class

$$
P=[(a(u), v-b(u))],
$$

where $a$ and $b$ are polynomials in $k[u]$ such that $a(u)=\prod_{i}\left(u-u_{i}\right)$ and $v_{i}=b\left(u_{i}\right)$ for all $i$. In this guise, addition of points $P$ and $Q$ is an ideal product, followed by a reduction algorithm to produce a unique "reduced" ideal representing $P+Q$. Cantor [1] provides algorithms to carry out these operations.

Algorithm 1. Given a semi-reduced representative $(a(u), v-b(u))$ for a point $P$ on the Jacobian of a hyperelliptic curve $X: v^{2}=f(u)$, returns the reduced representative of $P$.

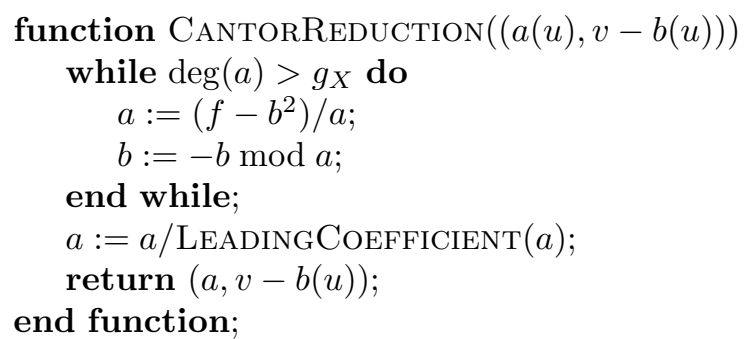

Each iteration of Algorithm 1 replaces $a$ with a polynomial of degree $\max \left(2 g_{X}+\right.$ $1-\operatorname{deg}(a), \operatorname{deg}(a)-2)$. It follows that Algorithm 1 will produce a reduced representative for the ideal class $[(a(u), v-b(u))]$ after $\left\lceil\left(\operatorname{deg}(a)-g_{X}\right) / 2\right\rceil$ iterations.

\section{$3 \quad$ Explicit Endomorphisms}

Let $C$ be a curve with an automorphism $\zeta$, and let $\pi: C \rightarrow X$ be a covering of $X$. We have two coverings, $\pi$ and $\pi \circ \zeta$, from $C$ to $X$; together, they induce a map $\eta$ of divisors

$$
\eta:=(\pi \circ \zeta)_{*} \pi^{*}: \operatorname{Div}(X) \rightarrow \operatorname{Div}(X)
$$

where

$$
\pi^{*}([P])=\sum_{Q \in \pi^{-1}(P)} e_{\pi}(Q)[Q] \text { and }(\pi \circ \zeta)_{*}([Q])=[\pi(\zeta(Q))] .
$$

This map on divisors induces an endomorphism of the $\operatorname{Jacobian} \operatorname{Jac}(X)$, which we also denote $\eta$.

In our constructions, we take $\pi$ to be the quotient by an involution $\sigma$ of $C$, so that $\pi$ is a degree- 2 covering, and $\pi=\pi \circ \sigma$. Thus

$$
\pi^{*}([P])=[Q]+[\sigma(Q)]
$$

for any point $Q$ in $\pi^{-1}(P)$. We will take $\zeta$ to be an automorphism of $C$ of prime order $p$, such that $\langle\zeta, \sigma\rangle$ is a dihedral subgroup of the automorphism group of $C$ : that is, $\sigma \zeta=\zeta^{-1} \sigma$. The following proposition describes the resulting endomorphism $\eta=(\pi \circ \zeta)_{*} \circ \pi^{*}$. 
Proposition 2. Let $C$ be a curve with an involution $\sigma$ and an automorphism $\zeta$ of prime order $p$ such that $\sigma \zeta=\zeta^{-1} \sigma$. Let $\pi: C \rightarrow X:=C /\langle\sigma\rangle$ be the quotient of $C$ by the action of $\sigma$, and let $\eta:=(\pi \circ \zeta)_{*} \circ \pi^{*}$ be the endomorphism of $\operatorname{Jac}(X)$ induced by $\zeta$. The subring $\mathbb{Z}[\eta]$ of $\operatorname{End}(\operatorname{Jac}(X))$ is isomorphic to $\mathbb{Z}\left[\zeta_{p}+\zeta_{p}^{-1}\right]$, where $\zeta_{p}$ is a primitive $p^{\text {th }}$ root of unity over $\mathbb{Q}$.

Proof. The subring $\mathbb{Z}\left[\zeta_{*}+\zeta_{*}^{-1}\right]$ of $\operatorname{Jac}(C)$ is isomorphic to $\mathbb{Z}\left[\zeta_{p}+\zeta_{p}^{-1}\right]$, since $p$ is prime. The statement follows upon noting that the following diagram commutes.

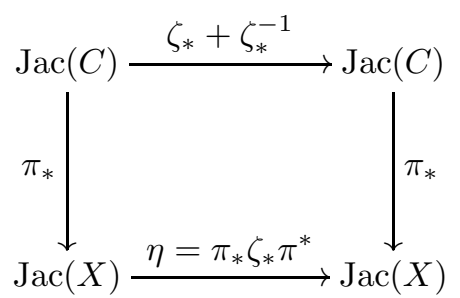

To see this, observe that for any $Q$ in $\operatorname{Jac}(C)$ we have

$$
\begin{aligned}
\eta\left(\pi_{*}(Q)\right) & =\pi_{*} \zeta_{*} \pi^{*} \pi_{*}(Q) \\
& =\pi_{*} \zeta_{*}\left(1+\sigma_{*}\right)(Q) \\
& =\pi_{*}\left(\zeta_{*}+\sigma_{*} \zeta_{*}^{-1}\right)(Q) \\
& =\pi_{*}\left(\zeta_{*}+\zeta_{*}^{-1}\right)(Q),
\end{aligned}
$$

since $\pi^{*} \pi_{*}=\left(1+\sigma_{*}\right)$ and $\pi_{*} \sigma_{*}=\pi_{*}$. See also Ellenberg [4, $\S 2$ ].

Suppose $C, X, \pi, \zeta$ and $\eta$ are as in Proposition 2 Our aim is to give an explicit realization of the endomorphism $\eta$ of $\operatorname{Jac}(X)$, in the form of a map on ideal classes. To do this, we form the algebraic correspondence

$$
Z:=(\pi \times(\pi \circ \zeta))(C) \subset X \times X .
$$

Let $\pi_{1}$ and $\pi_{2}$ be the restrictions to $Z$ of the projections from $X \times X$ to its first and second factors, respectively; then $\eta=\left(\pi_{2}\right)_{*} \circ \pi_{1}^{*}$. We will give an affine model for $Z$ as the variety cut out by an ideal in $k\left[u_{1}, v_{1}, u_{2}, v_{2}\right] /\left(v_{1}^{2}-f\left(u_{1}\right), v_{2}^{2}-f\left(u_{2}\right)\right)$; for this model, the maps $\pi_{1}$ and $\pi_{2}$ are defined by $\pi_{i}\left(u_{1}, v_{1}, u_{2}, v_{2}\right)=\left(u_{i}, v_{i}\right)$.

Suppose that $Z$ is defined by an ideal $\left(v_{2}-v_{1}, E\left(u_{1}, u_{2}\right)\right)$, where $E$ is quadratic in $u_{1}$ and $u_{2}$ (this will be the case in each of our constructions). If $(u, v)$ is a generic point on $X$, then $\pi_{1}^{*}([(u, v)])$ is the effective divisor on $Z$ cut out by $\left(v_{2}-v, E\left(u, u_{2}\right)\right)$. Therefore, if $e_{1}$ and $e_{2}$ are the solutions in $\overline{k(u)}$ to the quadratic equation $E(u, x)=0$ in $x$, then

$$
\eta([(u, v)])=\left(\pi_{2}\right)_{*} \pi_{1}^{*}([(u, v)])=\left[\left(e_{1}, v\right)\right]+\left[\left(e_{2}, v\right)\right] .
$$

It remains to translate this description of the action of $\eta$ in terms of points into a map on ideal classes.

Suppose $[(a(u), v-b(u))]$ is a point on $\operatorname{Jac}(X)$. Extending the above, we have

$$
\begin{aligned}
\eta([(a(u), v-b(u))]) & =\left[\left(a\left(e_{1}\right), v-b\left(e_{1}\right)\right)\right]+\left[\left(a\left(e_{2}\right), v-b\left(e_{2}\right)\right)\right] \\
& =\left[\left(N(a), v-\frac{(f(u)+N(b))}{T(b)} \bmod N(a)\right],\right.
\end{aligned}
$$


where $N(a)=a\left(e_{1}\right) a\left(e_{2}\right), N(b)=b\left(e_{1}\right) b\left(e_{2}\right)$, and $T(b)=b\left(e_{1}\right)+b\left(e_{2}\right) \cdot{ }^{1}$ Since functions $T(a), N(b)$ and $T(b)$ are symmetric polynomials in $e_{1}$ and $e_{2}$, we can write each as a polynomial in the elementary symmetric functions $e_{1}+e_{2}$ and $e_{1} e_{2}$. Moreover, $e_{1}+e_{2}$ and $e_{1} e_{2}$ are elements of $k(u)$ : if $E(u, x)=E_{2}(u) x^{2}+$ $E_{1}(u) x+E_{0}(u)$, then $e_{1}+e_{2}=-E_{1} / E_{2}$ and $e_{1} e_{2}=E_{0} / E_{2}$.

Definition 3. For any polynomial a $(x)$ over $k$, we define $T(a)=a\left(e_{1}\right)+a\left(e_{2}\right)$ and $N(a)=a\left(e_{1}\right) a\left(e_{2}\right)$, and for $i, j \geq 0$ we define

$$
t_{i}:=e_{1}^{i}+e_{2}^{i}, n_{i}:=\left(e_{1} e_{2}\right)^{i} \quad \text { and } \quad n_{i, j}:=e_{1}^{i} e_{2}^{j}+e_{1}^{j} e_{2}^{i} .
$$

Note that $t_{i}$ and $n_{i j}$ are elements of $k(u)$ and that

$$
T\left(\sum_{i=0}^{g_{X}} a_{i} x^{i}\right)=\sum_{i=0}^{g_{X}} a_{i} t_{i},
$$

and

$$
N\left(\sum_{i=0}^{g_{X}} a_{i} x^{i}\right)=\sum_{i=0}^{g_{X}} \sum_{i=0}^{g_{X}} a_{i} a_{j} n_{i, j} .
$$

The following elementary lemma provides simple recurrences for the construction of the sequences $\left\{t_{i}\right\}$ and $\left\{n_{i, j}\right\}$.

Lemma 4. The elements $t_{i}, n_{i}$ and $n_{i, j}$ satisfy the following recurrences:

1. $n_{i+1}=\left(e_{1} e_{2}\right) n_{i}$ for $i \geq 0$, with $n_{0}:=1$;

2. $t_{i+1}=\left(e_{1}+e_{2}\right) t_{i}-\left(e_{1} e_{2}\right) t_{i-1}$ for $i \geq 1$, with $t_{0}=2$ and $t_{1}=\left(e_{1}+e_{2}\right)$;

3. $n_{i, i}=n_{i}$ and $n_{i, j}=n_{i} t_{j-i}$ for $i \geq 0$ and $j>i$.

Equations (11) and (2) above express $T$ and $N$ in terms of the functions $t_{i}$ and $n_{i, j}$, which depend only upon $t_{1}$ and $n_{1}$ by Lemma 4 Thus, given $t_{1}=e_{1}+e_{2}$ and $n_{1}=n_{1,1}=e_{1} e_{2}$, the recurrences of Lemma 4 give a simple and fast algorithm for computing the maps $T$ and $N$. If we further assume that $T$ and $N$ will only be evaluated at polynomials $a$ and $b$ from reduced ideal class representatives $(a(u), v-b(u))$, then we need only compute the $t_{i}$ and $n_{i, j}$ for $0 \leq i \leq j \leq g_{X}$.

Algorithm 5. Given functions $t_{1}$ and $n_{1}$ in $k(u)$, together with the genus $g_{X}$ of a curve $X$, returns the maps $T$ and $N$ of Definition 3

function Rationalmaps $\left(t_{1}, n_{1}, g_{X}\right)$

$$
\begin{aligned}
& n_{0}:=1 ; \\
& t_{0}:=2 ; \\
& \text { for } i \text { in }\left[1, \ldots, g_{X}\right] \text { do } \\
& \quad n_{i+1}:=n_{1} n_{i} ; \\
& \quad t_{i+1}:=t_{1} t_{i}-n_{1} t_{i-1} ;
\end{aligned}
$$

\footnotetext{
${ }^{1}$ The modular inversion of $T(b)$ should be carried out after clearing denominators and removing common factors from $N(a), T(b)$, and $f(u)+N(b)$ (generically, $N(a)$ and $T(b)$ are coprime). Proposition [6 below makes this precise.
} 


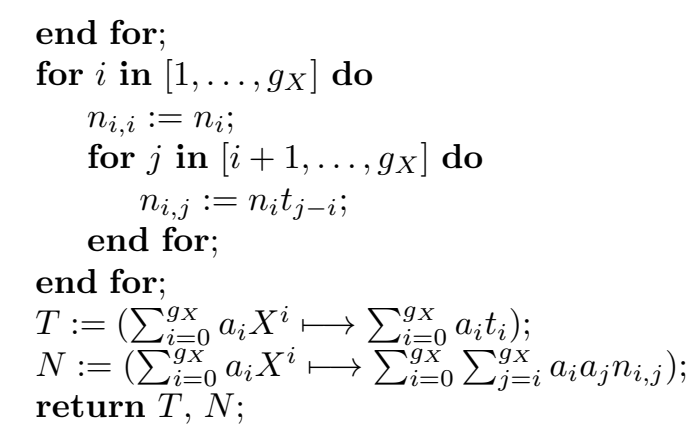

\section{end function;}

The following proposition shows that the maps $T$ and $N$ may be used to compute $\eta([(a(u), v-b(u))])$ for all points $[(a(u), v-b(u))]$ of $\operatorname{Jac}(X)$.

Proposition 6. Let $\eta$ be the endomorphism of $\operatorname{Jac}(X)$ induced by a correspondence $V\left(v_{2}-v_{1}, E_{2}\left(u_{1}\right) u_{2}^{2}+E_{1}\left(u_{1}\right) u_{2}+E_{0}\left(u_{1}\right)\right)$ on $X \times X$; set $t_{1}=-E_{1} / E_{2}$ and $n_{1}=E_{0} / E_{2}$, and let $T$ and $N$ be the maps of Definition 3 . If $(a(u), v-b(u))$ is the reduced representative of a point $P$ of $\operatorname{Jac}(X)$, then $\eta(P)$ is represented by

$$
\left(\frac{E_{2}^{g_{X}} N(a)}{G}, v-\left(\frac{(f+N(b)) / G}{T(b) / G} \bmod \frac{E_{2}^{g X} N(a)}{G}\right)\right),
$$

where $G=\operatorname{gcd}\left(E_{2}^{g_{X}} N(a), E_{2}^{g_{X}} T(b)\right)$. Algorithm 1 computes the reduced representative of $\eta(P)$ after at most $\left\lceil g_{X} / 2\right\rceil$ iterations of its main loop.

Proof. We have

$$
\begin{aligned}
\eta([(a(u), v-b(u))]) & =\left[\left(a\left(e_{1}\right), v-b\left(e_{1}\right)\right)\left(a\left(e_{2}\right), v-b\left(e_{2}\right)\right)\right] \\
& =\left[\left(N(a), v^{2}-T(b) v+N(b)\right)\right] \\
& =\left[\left(E_{2}^{g_{X}}\right)(N(a), T(b) v-(f+N(b)))\right] . \\
& =\left[\left(E_{2}^{g_{X}} N(a), E_{2}^{g_{X}} T(b) v-E_{2}^{g_{X}}(f+N(b))\right)\right] .
\end{aligned}
$$

It is easily verified that $E_{2}^{g X} N(a), E_{2}^{g X} T(b)$ and $E_{2}^{g X}(f+N(b))$ are polynomials, and that if $G=\operatorname{gcd}\left(E_{2}^{g_{X}} N(a), E_{2}^{g_{X}} T(b)\right)$, then $G$ also divides $E_{2}^{g_{X}}(f+N(b))$. Therefore

$$
\begin{aligned}
\eta([(a(u), v-b(u))]) & \left.=\left[(G)\left(E_{2}^{g_{X}} N(a) / G, E_{2}^{g_{X}} T(b) v / G-E_{2}^{g_{X}}(f+N(b)) / G\right)\right)\right] \\
& =\left[\left(E_{2}^{g_{X}} N(a) / G, E_{2}^{g_{X}} T(b) v / G-E_{2}^{g_{X}}(f+N(b)) / G\right)\right] \\
& =\left[\left(E_{2}^{g_{X}} N(a) / G, v-I \cdot E_{2}^{g_{X}}(f+N(b)) / G\right)\right],
\end{aligned}
$$

where $I$ denotes the inverse of $E_{2}^{g_{X}}(f+N(b)) / G$ modulo $E_{2}^{g_{X}} N(a) / G$, proving the first claim. Now, if $(a(u), v-b(u))$ is the reduced representative of $P$, then $\operatorname{deg}(a) \leq g_{X}$, so the degree of $E_{2}^{g_{X}} N(a)$ is at most $2 g_{X}$. After each iteration of Algorithm 1he degree of $a$ becomes $\max \left(2 g_{X}+1-\operatorname{deg}(a), \operatorname{deg}(a)-2\right)$, and the algorithm terminates when $\operatorname{deg}(a) \leq g_{X}$; this occurs after $\left\lceil g_{X} / 2\right\rceil$ iterations.

The following algorithm applies Proposition [ to compute the image of a point of $\operatorname{Jac}(X)$ under $\eta$. This gives an explicit realization of $\eta$ as a map on ideal classes. 
Algorithm 7. Given a point $P$ on the Jacobian of a curve $X: v^{2}=f(u)$ and rational maps $T$ and $N$ derived for an endomorphism $\eta$ of $\operatorname{Jac}(X)$ using Algorithm [5 returns the reduced ideal class representative of $\eta(P)$.

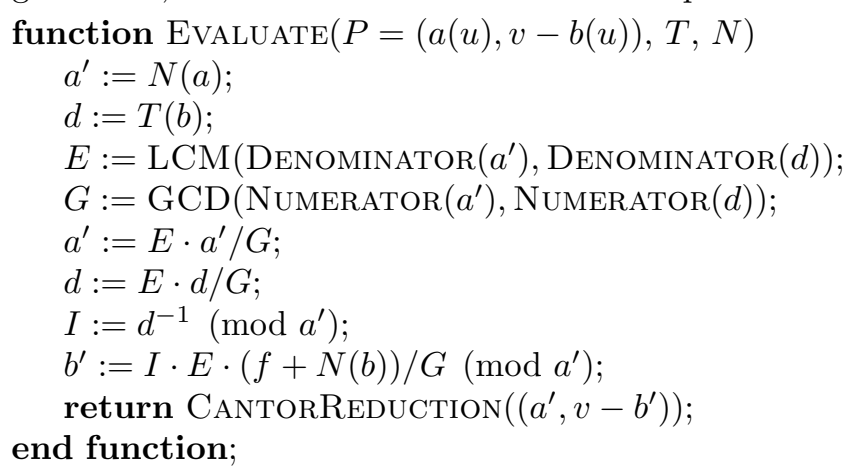

Remark 8. In the families of curves described below in Sections 4 and 5 below, $T$ and $N$ are polynomial maps, and we may take $E=1$ in Algorithm 7

\section{Applications I: Curves with Artin-Schreier Covering}

In this section we construct a family of curves $X_{p}$ in one free parameter $t$ for each prime $p \geq 5$, and determine explicit endomorphisms deriving from a cover by the Artin-Schreier curve defined over $\mathbb{F}_{p}$ by

$$
C_{p}: y^{p}-y=x+\frac{t}{x}
$$

The eigenvalues of Frobenius in this family are described by classical Kloosterman sums [21].

An analogous family $y^{2}=x^{p}-x+t$ was described by Duursmaa and Sakurai [3], for which the automorphism $x \mapsto x+1$ was proposed for efficient scalar multiplication. In constrast to our family, every member of this family is isomorphic over a base extension to the supersingular curve $y^{2}=x^{p}-x$.

\subsection{Construction of the Artin-Schreier Covering}

The curve $C_{p}$ has automorphisms $\zeta$ (of order $p$ ) and $\sigma$ (of order 2), defined by

$$
\zeta(x, y)=(x, y+1) \quad \text { and } \quad \sigma(x, y)=(-t / x,-y) .
$$

Let $X_{p}$ be the quotient of $C_{p}$ by $\langle\sigma\rangle$, with affine model

$$
X_{p}: v^{2}=f(u)=u\left(u^{(p-1) / 2}-1\right)^{2}-4 t .
$$

The quotient map $\pi: C_{p} \rightarrow X_{p}$ is a covering of degree 2 , sending $(x, y)$ to $(u, v)=\left(y^{2}, x-t / x\right)$. Observe that $X_{p}$ is a family of curves of genus $(p-1) / 2$. 
The automorphism $\zeta$ of $C_{p}$ induces an endomorphism $\eta:=(\pi \circ \zeta)_{*} \pi^{*}$ on $\operatorname{Jac}\left(X_{p}\right)$, whose minimal polynomial equals that of $\eta_{p}=\zeta_{p}+\zeta_{p}^{-1} \in \mathbb{C}$. The endomorphism $\eta$ is induced by the correspondence $Z:=(\pi \circ \zeta \times \pi)\left(C_{p}\right)$ on $X_{p} \times X_{p}$, for which we may directly compute an affine model

$$
Z=V\left(v_{2}-v_{1}, u_{2}^{2}+u_{1}^{2}-2 u_{1} u_{2}-2 u_{2}-2 u_{1}+1\right) .
$$

Setting $t_{1}:=2(u+1)$ and $n_{1}:=(u-1)^{2}$ and applying Algorithm [5 we obtain polynomial maps $T$ and $N$ such that $\eta$ is realized by $P \mapsto \operatorname{Evaluate}(P, T, N)$, using Algorithm 7 The first few $t_{i}$ and $n_{i, j}$ derived in Algorithm [5 are given in Table 1 below.

Proposition 9. The Jacobian $\operatorname{Jac}\left(C_{p}\right)$ is isogenous to $\operatorname{Jac}\left(X_{p}\right)^{2}$, and its endomorphism ring contains an order in $\mathbb{M}_{2}\left(\mathbb{Q}\left(\eta_{p}\right)\right)$.

Proof. The automorphisms $\zeta$ and $\sigma$ determine a homomorphic image of the group algebra $A=\mathbb{Q}[\langle\zeta, \sigma\rangle]$ in $\operatorname{End}^{\circ}\left(\operatorname{Jac}\left(C_{p}\right)\right)$. But $A$ is a semisimple algebra of dimension $2 p$ over $\mathbb{Q}$, whose simple quotients are of dimensions 1,1 , and $2 \varphi(p)$. Moreover, $\zeta+\zeta^{-1}$ is in the centre of $A$ and generates a subring isomorphic to $\mathbb{Q} \times \mathbb{Q}\left(\eta_{p}\right)$. Since $\zeta$ and $\sigma$ do not commute, it follows that the latter algebra is isomorphic to $\mathbb{M}_{2}\left(\mathbb{Q}\left(\eta_{p}\right)\right)$.

Let $e_{1}$ and $e_{2}$ be the central idempotents associated to the quotients of dimensions 1 . On each associated abelian variety $e_{i} \operatorname{Jac}\left(C_{p}\right)$, the automorphism $\zeta$ acts trivially, thus maps through the Jacobian of the genus 0 quotient $C_{p} /\langle\zeta\rangle$; it follows that the image of $A$ in $\operatorname{End}^{\circ}\left(\operatorname{Jac}\left(C_{p}\right)\right)$ is isomorphic to $\mathbb{M}_{2}\left(\mathbb{Q}\left(\eta_{p}\right)\right)$.

Let $\epsilon_{1}=1+\sigma$ and $\epsilon_{2}=1-\sigma$. Noting that

$$
\epsilon_{i}^{2}=2 \epsilon_{i}, \quad \epsilon_{1} \epsilon_{2}=0, \text { and } \epsilon_{1}+\epsilon_{2}=2,
$$

we let $A_{1}=\epsilon_{1 *} \operatorname{Jac}\left(C_{p}\right)$ and $A_{2}=\epsilon_{2 *} \operatorname{Jac}\left(C_{p}\right)$ be subabelian varieties of $\operatorname{Jac}\left(C_{p}\right)$ such that $\operatorname{Jac}\left(C_{p}\right)=A_{1}+A_{2}$, and $A_{1} \cap A_{2}$ is finite. Since $\zeta-\zeta^{-1}$ determines an isogeny $\psi=\zeta_{*}-\zeta_{*}^{-1}$ of $\operatorname{Jac}\left(C_{p}\right)$ to itself, the relation

$$
\left(\zeta-\zeta^{-1}\right) \epsilon_{1}=\epsilon_{2}\left(\zeta-\zeta^{-1}\right)
$$

implies that $\psi\left(A_{1}\right)=\epsilon_{2 *} \psi\left(\operatorname{Jac}\left(C_{p}\right)\right)=A_{2}$, so that $A_{1}$ and $A_{2}$ are isogenous. But $\pi_{*}$ is an isogeny of $A_{1}$ to $\operatorname{Jac}\left(X_{p}\right)$, whence $\operatorname{Jac}\left(C_{p}\right) \sim \operatorname{Jac}\left(X_{p}\right)^{2}$.

Corollary 10. The Jacobian $\operatorname{Jac}\left(X_{p}\right)$ has a rational p-torsion point. In particular, $\operatorname{Jac}\left(X_{p}\right)$ is not a supersingular abelian variety.

Proof. The curve $C_{p}$ has two rational points fixed by $\zeta$, whose difference determines a point in $\operatorname{ker}\left(1-\zeta_{*}\right)$. But

$$
(1-\zeta)\left(1-\zeta^{2}\right) \cdots\left(1-\zeta^{p-1}\right)=p,
$$

so $\operatorname{ker}\left(1-\zeta_{*}\right)$ is contained in $\operatorname{Jac}\left(X_{p}\right)[p]$. If $\chi(T)$ and $\xi(T)$ are the characteristic polynomials of Frobenius on $\operatorname{Jac}\left(C_{p}\right)$ and $\operatorname{Jac}\left(X_{p}\right)$, respectively, then $\chi(T)=$ $\xi(T)^{2}$. Since $\left|\operatorname{Jac}\left(C_{p}\right)(k)\right|=\chi(1)$ is divisible by $p$, so is $\left|\operatorname{Jac}\left(X_{p}\right)(k)\right|=\xi(1)$.

Remark 11. In fact, it is possible to show that the $p$-rank of $\operatorname{Jac}\left(X_{p}\right)$ is exactly equal to 1 , so the Jacobians are neither ordinary nor supersingular. 
Table 1. Artin-Schreier covers: $t_{i}$ and $n_{i, j}$ for $0 \leq i \leq j \leq 3$.

\begin{tabular}{|c|l|}
\hline$t_{0}$ & 2 \\
$t_{1}$ & $2(u+1)$ \\
$t_{2}$ & $2\left(u^{2}+6 u+1\right)$ \\
$t_{3}$ & $2\left(u^{3}+15 u^{2}+15 u+1\right)$ \\
\hline$n_{0,0}$ & 1 \\
$n_{0,1}$ & $2(u+1)$ \\
$n_{0,2}$ & $2\left(u^{2}+6 u+1\right)$
\end{tabular}$\left[\begin{array}{l|l|l|}n_{0,3} & 2\left(u^{3}+15 u^{2}+15 u+1\right) \\
n_{1,1} & (u-1)^{2} \\
n_{1,2} & 2(u-1)^{2}(u+1) \\
n_{1,3} & 2(u-1)^{2}\left(u^{2}+6 u+1\right) \\
n_{2,2} & (u-1)^{4} \\
n_{2,3} & 2(u-1)^{4}(u+1) \\
n_{3,3} & (u-1)^{6} \\
\hline\end{array}\right.$

\subsection{Hyperelliptic Curves of Genus 2 with Real Multiplication by $\eta_{5}$}

For $p=5$, the construction above yields a one-parameter family of genus 2 hyperelliptic curves defined by

$$
X_{5}: v^{2}=f_{5}(u)=u\left(u^{2}-1\right)^{2}+t,
$$

whose Jacobian has endomorphism ring containing $\mathbb{Z}\left[\eta_{5}\right] \cong \mathbb{Z}[x] /\left(x^{2}+x-1\right)$.

Each point $P$ of $\operatorname{Jac}\left(X_{5}\right)$ may be represented by an ideal $(a(u), v-b(u))$ with $a$ and $b$ of degrees 2 and 1 respectively: hence, suppose $a(u)=a_{2} u^{2}+a_{1} u+a_{0}$ and $b(u)=b_{1} u+b_{0}$. Applying Algorithm [ we see that

$$
\begin{aligned}
N(a) & =a_{2}^{2} n_{2,2}+a_{2} a_{1} n_{1,2}+a_{1}^{2} n_{1,1}+a_{2} a_{0} n_{0,2}+a_{1} a_{0} n_{0,1}+a_{0}^{2} n_{0,0}, \\
N(b) & =b_{1}^{2} n_{1,1}+b_{1} b_{0} n_{0,1}+b_{0}^{2} n_{0,0}, \text { and } \\
T(b) & =2 b_{1}(u+1)+2 b_{0},
\end{aligned}
$$

with the $n_{i, j}$ as in Table 1 The endomorphism $\eta$ is then explicitly realized by $\eta: P \mapsto \operatorname{Evaluate}(P, T, N)$, using Algorithm $\mathbf{7}$

Remark 12. The Igusa invariants of the curve $X_{5}$ determine the weighted projective point $\left(J_{2}: J_{4}: J_{6}: J_{8}: J_{10}\right)=\left(3: 2: 0: 4: 4 t^{2}\right)$. In particular, the curves determine a one-dimensional subvariety of the moduli space of genus 2 curves.

\subsection{Hyperelliptic Curves of Genus 3 with Real Multiplication by $\eta_{7}$}

For $p=7$, we derive a family of genus 3 hyperelliptic curves

$$
X_{7}: v^{2}=u\left(u^{3}-1\right)^{2}+3 t
$$

and an endomorphism $\eta$ of $\operatorname{Jac}\left(X_{7}\right)$ with $\mathbb{Z}[\eta] \cong \mathbb{Z}\left[\zeta_{7}+\zeta_{7}^{-1}\right]$ by Proposition 2 Applying Algorithm [5 we derive polynomial maps $T$ and $N$, which we use with Algorithm 7 to realize $\eta$ as $\eta: P \mapsto \operatorname{Evaluate}(P, T, N)$.

\section{Applications II: Curves with Cyclotomic Covering}

In this section we develop explicit endomorphisms for the one dimensional families of hyperelliptic curves with real multiplication based on cyclotomic coverings, as defined in Tautz, Top, and Verberkmoes [20]. 


\subsection{Construction of the Cyclotomic Covering}

Let $n \geq 2$, and let $\rho_{n}$ and $\rho_{2 n}$ be primitive $n^{\text {th }}$ and $2 n^{\text {th }}$ roots of unity over $k$ such that $\rho_{2 n}^{2}=\rho_{n}$; also set $\tau_{n}=\rho_{n}+\rho_{n}^{-1}$. Consider the family of hyperelliptic curves of genus $n$ over $k$ in one free parameter $t$ defined by

$$
C_{n}: y^{2}=x\left(x^{2 n}+t x^{n}+1\right) .
$$

The curve $C_{n}$ has an automorphism $\zeta$ of order $2 n$ and an involution $\sigma$, defined by

$$
\zeta:(x, y) \longmapsto\left(\rho_{n} x, \rho_{2 n} y\right) \quad \text { and } \quad \sigma:(x, y) \longmapsto\left(x^{-1}, x^{-(n+1)} y\right),
$$

respectively; note that $\zeta^{n}$ is the hyperelliptic involution $(x, y) \mapsto(x,-y)$. We define $X_{n}:=C_{n} /\langle\sigma\rangle$ to be the quotient of $C_{n}$ by the action of $\sigma$. The curve $X_{n}$ has an an affine model

$$
X_{n}: v^{2}=f_{n}(u)=D_{n}(u, 1)+t,
$$

where $D_{n}(u, 1)$ is the $n^{\text {th }}$ Dickson polynomial of the first kind with parameter ${ }^{2} 1$, defined recursively by

$$
D_{n}(u, 1)=u D_{n-1}(u, 1)-D_{n-2}(u, 1)
$$

for $n \geq 2$, with $D_{0}(u, 1)=2$ and $D_{1}(u, 1)=u$. Dickson polynomials and their properties are described in [12]; for our purposes, it is enough to know that

$$
D_{n}\left(u+u^{-1}, 1\right)=u^{n}+u^{-n}
$$

(this is easily verified by induction), which further implies

$$
D_{n m}(u, 1)=D_{n}\left(D_{m}(u, 1), 1\right) .
$$

Remark 13. When $n$ is odd, our curves $C_{n}$ and $X_{n}$ coincide with the curves $\mathcal{D}_{n}$ and $\mathcal{C}_{n}$ of [20]; for even $n$, our families instead coincide with the curves described in the remark of [20, page 1058].

The quotient projection $\pi: C_{n} \rightarrow X_{n}$ is a covering of degree 2. Equation (4) above shows that it is defined by

$$
\pi:(x, y) \longmapsto(u, v)=\left(x+x^{-1}, x^{-(n+1 / 2)} y\right) .
$$

The automorphism $\zeta$ of $C_{n}$ induces an endomorphism $\eta=(\pi \circ \zeta)_{*} \circ \pi^{*}$ of $\operatorname{Jac}\left(X_{n}\right)$. If $n$ is prime, then Proposition 2 implies that $\mathbb{Z}[\eta] \cong \mathbb{Z}\left[\zeta_{n}+\zeta_{n}^{-1}\right]$, where $\zeta_{n}$ is an $n^{\text {th }}$ root of unity over $\mathbb{Q}$.

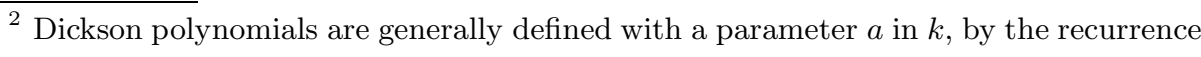$$
D_{n}(u, a)=u D_{n-1}(u, a)-a D_{n-2}(u, a) .
$$

It is easily shown that the curve defined by $v^{2}=D_{n}(u, a)+t$ for any nonzero $a$ is a twist of $X_{n}$. When $a=0$, we obtain a one-dimensional family of curves with complex multiplication by $\mathbb{Z}\left[\zeta_{n}\right]$; these curves are described in [16] $\left.\$ 6.4\right]$.
} 
The endomorphism $\eta$ is induced by the correspondence $Z:=(\pi \circ \zeta \times \pi)\left(C_{n}\right)$ on $X_{n} \times X_{n}$, for which we directly compute an affine model

$$
Z=V\left(v_{2}-v_{1}, u_{2}^{2}+u_{1}^{2}-\tau_{n} u_{1} u_{2}+\tau_{n}^{2}-4\right) .
$$

Setting $t_{1}:=\tau_{n} u$ and $n_{1}:=u^{2}+\tau_{n}^{2}-4$, we apply Algorithm $[5$ to obtain maps $T: k[u] \rightarrow k[u]$ and $N: k[u] \rightarrow k[u]$ such that the endomorphism $\eta$ is realized by $P \mapsto \operatorname{Evaluate}(P, T, N)$, using Algorithm $\mathbf{7}$ The first few $t_{i}$ and $n_{i, j}$ derived in Algorithm 5 are given in Table 2 below.

Table 2. Cyclotomic covers: $t_{i}$ and $n_{i, j}$ for $0 \leq i \leq j \leq 3$

\begin{tabular}{|c|l|l|l|}
\hline$t_{0}$ & 2 \\
$t_{1}$ & $\tau_{n} u$ \\
$t_{2}$ & $\left(\tau_{n}^{2}-2\right) u^{2}-2\left(\tau_{n}^{2}-4\right)$ \\
$t_{3}$ & $\tau_{n}\left(\tau_{n}^{2}-3\right) u^{3}-3 \tau_{n}\left(\tau_{n}^{2}-4\right) u$ \\
\hline$n_{0,0}$ & 1 \\
$n_{0,1}$ & $\tau_{n} u$ \\
$n_{0,2}$ & $\left(\tau_{n}^{2}-2\right) u^{2}-2\left(\tau_{n}^{2}-4\right)$
\end{tabular}$\left[\begin{array}{ll|l}n_{0,3} & \tau_{n}\left(\left(\tau_{n}^{2}-3\right) u^{2}-3\left(\tau_{n}^{2}-4\right)\right) u \\
n_{1,1} & u^{2}+\tau_{n}^{2}-4 \\
n_{1,2} & \tau_{n}\left(u^{2}+\tau_{n}^{2}-4\right) u \\
n_{1,3} & \left(\tau^{2}-2\right) u^{4}+\left(\tau^{2}-4\right)^{2}\left(u^{2}-2\right) \\
n_{2,2} & \left(u^{2}+\tau_{n}^{2}-4\right)^{2} \\
n_{2,3} & \left.\tau_{n}\left(u^{2}+\tau_{n}^{2}-4\right)^{2}\right) u \\
n_{3,3} & \left(u^{2}+\tau_{n}^{2}-4\right)^{3} \\
\hline\end{array}\right.$

The elliptic curve $C_{1}: y^{2}=x\left(x^{2}+t x+1\right)$ is obviously covered by $C_{n}$, and is therefore a factor of $\operatorname{Jac}\left(C_{n}\right)$. The following analogue of Theorem 9 holds for this cyclotomic family, and is proved similarly.

Proposition 14. The Jacobian $\operatorname{Jac}\left(C_{n}\right)$ is isogenous to $C_{1} \times \operatorname{Jac}\left(X_{n}\right)^{2}$ for $n$ prime, and its endomorphism ring contains an order in $\mathbb{Q} \times \mathbb{M}_{2}\left(\mathbb{Q}\left(\eta_{n}\right)\right)$.

Remark 15. If $n$ is a prime other than 5, then [20, Corollary 6] implies that $\operatorname{Jac}\left(X_{n}\right)$ is absolutely simple for general values of $t$ over a field of characteristic 0 . For $n=5$, we find that the condition of Stoll [18] (see [5 §14.4]) for $\operatorname{Jac}\left(X_{5}\right)$ to be absolutely simple is satisfied by $X_{5}$ with $t=1$ at $p=11$. Conversely, if $n=p m$, for $p>2$ and $m>1$, then identity (5) above gives a covering $X_{n} \rightarrow X_{p}$ of degree $m$, defined by $(u, v) \mapsto\left(D_{m}(u, 1), v\right)$. It follows that $\operatorname{Jac}\left(X_{n}\right)$ has a factor isogenous to $\mathrm{Jac}\left(X_{p}\right)$, and so is not simple.

\subsection{Hyperelliptic Curves of Genus 2 with Real Multiplication by $\boldsymbol{\eta}_{5}$}

Consider the case $n=5$. Equation (3) shows that $D_{5}(u, 1)=u^{5}-5 u^{3}+5 u$, so the curve $X_{5}=C_{5} /\langle\sigma\rangle$ is the curve of genus 2 defined by the affine model

$$
X_{5}: v^{2}=f_{5}(u)=u^{5}-5 u^{3}+5 u+t .
$$

Each point on $\operatorname{Jac}\left(X_{5}\right)$ has a representative in the form $(a(u), v-b(u))$, with $\operatorname{deg} a=2$ and $\operatorname{deg} b=1$; so suppose $a(u)=a_{2} u^{2}+a_{1} u+a_{0}$ and $b(u)=b_{1} u+b_{0}$. Applying Algorithm [5 we obtain maps $T$ and $N$ such that

$$
\begin{aligned}
N(a)= & a_{2}^{2} u^{4}+a_{2} a_{1} \tau_{5} u^{3}+\left(2 a_{2}^{2}\left(\tau_{5}^{2}-4\right)+a_{1}^{2}+a_{2} a_{0}\left(\tau_{5}^{2}-2\right)\right) u^{2} \\
& +a_{1}\left(a_{2}\left(\tau_{5}^{2}-4\right)+a_{0}\right) \tau_{5} u+\left(\left(\tau_{5}^{2}-4\right)\left(a_{2}^{2}\left(\tau_{5}^{2}-4\right)+a_{1}^{2}-2 a_{2} a_{0}\right)+a_{0}^{2}\right), \\
N(b)= & b_{1}^{2} u^{2}+b_{1} b_{0} \tau_{5} u+b_{1}^{2}\left(\tau_{5}^{2}-4\right)+b_{0}^{2}, \text { and } \\
T(b)= & \tau_{5} b_{1} u+2 b_{0} .
\end{aligned}
$$


The endomorphism $\eta$ is then explicitly realized by $\eta: P \mapsto \operatorname{Evaluate}(P, T, N)$, using Algorithm $\mathbf{7}$

Remark 16. The weighted projective Igusa invariants of the generic curve are:

$$
\left(140: 550: 640 t^{2}-60: 22400 t^{2}-77725: 256 t^{4}-2048 t^{2}+4096\right) \text {. }
$$

In particular, this family corresponds to a one-dimensional subvariety in the moduli space.

\subsection{Hyperelliptic Curves of Genus 3 with Real Multiplication by $\boldsymbol{\eta}_{\mathbf{7}}$}

In the case $n=7$, we derive a family of curves

$$
X_{7}: v^{2}=u^{7}-7 u^{5}+14 u^{3}-7 u+t,
$$

and an endomorphism $\eta$ of $\operatorname{Jac}\left(X_{7}\right)$ with $\mathbb{Z}[\eta] \cong \mathbb{Z}\left[\zeta_{7}+\zeta_{7}^{-1}\right]$ by Proposition 2 Applying Algorithm 5 we derive polynomial maps $T$ and $N$, which we may then use with Algorithm 7 to realize $\eta$ as $\eta: P \mapsto \operatorname{Evaluate}(P, T, N)$.

\section{Applications III: Curves From Elliptic Coverings}

In 14, Mestre constructs a series of two dimensional families of hyperelliptic curves with explicit real endomorphisms, which are similarly realized by explicit correspondences. For the case $\eta_{5}$, Takashima 19] independently developed an explicit algorithm and complexity analysis for two and three dimensional families ${ }^{3}$ referred to as Mestre-Hashimoto and Brumer-Hashimoto (see [8]).

\subsection{Hyperelliptic Curves of Genus 2 with Real Multiplication by $\boldsymbol{\eta}_{\mathbf{5}}$}

Let $s$ and $t$ be free parameters, and consider the family of curves defined by

$$
X_{5}: v^{2}=f_{5}(u)=u^{4}(u-s)-s(u+1)(u-s)^{3}+s^{3} u^{3}-t u^{2}(u-s)^{2} .
$$

Mestre shows that $\operatorname{Jac}\left(X_{5}\right)$ has an endomorphism $\eta$ satisfying $\eta^{2}+\eta-1=0$, induced by the correspondence $Z$ with affine model

$$
Z=V\left(v_{2}-v_{1}, u_{1}^{2} u_{2}^{2}+s(s-1) u_{1} u_{2}-s^{2}\left(u_{1}-u_{2}\right)+s^{3}\right) .
$$

We will derive an explicit form for $\eta$. Since $X_{5}$ is a curve of genus 2, each point of $\operatorname{Jac}\left(X_{5}\right)$ may be represented by an ideal $(a(u), v-b(u))$ with $a=a_{2} u^{2}+a_{1} u+a_{0}$ and $b=b_{1} u+b_{0}$. Setting $t_{1}=-s\left((s-1) u_{2}-s\right) / u_{2}^{2}$ and $n_{1}=s^{2}\left(u_{2}+s\right) / u_{2}^{2}$, we apply Algorithm $[5$ to derive maps $T$ and $N$ such that

$$
\begin{aligned}
N(a) & =a_{2}^{2} n_{2,2}+a_{2} a_{1} n_{1,2}+a_{1}^{2} n_{1,1}+a_{2} a_{0} a_{2} n_{0,2}+a_{1} a_{0} n_{0,1}+a_{0}^{2} n_{0,0}, \\
N(b) & =b_{1}^{2} n_{1,1}+b_{1} b_{0} n_{0,1}+b_{0}^{2} n_{0,0}, \text { and } \\
T(b) & =-b_{1} s((s-1) u-s) / u^{2}+2 b_{0},
\end{aligned}
$$

with the $n_{i, j}$ given in the table below.

\footnotetext{
3 The moduli of genus 2 curves with real multiplication by $\eta_{5}$ form a two dimensional subvariety of the moduli space of genus 2 curves, so this three dimensional family contains one dimensional fibres of geometrically isomorphic curves.
} 


\begin{tabular}{|l|l|l|l|}
\hline$n_{0,0}$ & 1 \\
$n_{0,1}$ & $-s((s-1) u-s) / u^{2}$ \\
$n_{0,2}$ & $s^{2}\left(((s-1) u-s)^{2}-2 u^{2}(u+s)\right) / u^{4}$
\end{tabular} \begin{tabular}{ll|l|}
$n_{1,1}$ & $s^{2}(u+s) / u^{2}$ \\
$n_{1,2}$ & $-s^{3}(u+s)((s-1) u-s) / u^{4}$ \\
$n_{2,2}$ & $s^{4}(u+s)^{2} / u^{4}$ \\
\hline
\end{tabular}

The endomorphism $\eta$ is then explicitly realized by $\eta: P \mapsto \operatorname{Evaluate}(P, T, N)$, using Algorithm 7

\subsection{Hyperelliptic Curves of Genus 3 with Real Multiplication by $\eta_{7}$}

Let $s$ and $t$ be free parameters, and consider the family of hyperelliptic genus 3 curves defined by

$$
X_{7}: v^{2}=f_{7}(u)=\phi_{7}(u)-t \psi_{7}(u)^{2}
$$

where $\psi_{7}(u):=u\left(u-s^{3}+s^{2}\right)\left(u-s^{2}+s\right)$ and

$$
\begin{aligned}
\phi_{7}(u):=u \psi_{7}(u)^{2} & +s(s-1)\left(s^{2}-s+1\right)\left(s^{3}+2 s^{2}-5 s+1\right) u^{5} \\
& -s^{3}(s-1)^{2}\left(6 s^{4}-11 s^{3}+12 s^{2}-11 s-1\right) u^{4} \\
& +s^{4}(s-1)^{3}\left(s^{2}-s-1\right)\left(s^{3}+2 s^{2}+6 s+1\right) u^{3} \\
& -s^{6}(s-1)^{4}(s+1)\left(3 s^{2}-5 s-3\right) u^{2} \\
& +s^{8}(s-1)^{5}\left(s^{2}-3 s-3\right) u+s^{10}(s-1)^{6} .
\end{aligned}
$$

Mestre shows that $\operatorname{Jac}\left(X_{7}\right)$ has an endomorphism $\eta$ satisfying $\eta^{3}+\eta^{2}-2 \eta-1=0$, induced by the correspondence $Z=V\left(v_{2}-v_{1}, E\right)$ on $X_{7} \times X_{7}$, where

$$
E=u_{1}^{2} u_{2}^{2}-s^{2}(s-1)\left(s^{2}-s-1\right) u_{1} u_{2}-s^{4}(s-1)^{2}\left(u_{1}+u_{2}\right)+s^{6}(s-1)^{3} .
$$

Since $X_{7}$ is a curve of genus 3, each point on $\operatorname{Jac}\left(X_{7}\right)$ may be represented by an ideal $(a(u), v-b(u))$, where $a$ and $b$ are polynomials of degree 3 and 2, respectively. Setting

$$
\begin{aligned}
t_{1} & =s^{2}(s-1)\left(\left(s^{2}-s-1\right) u+s^{2}(s-1)\right) / u^{2} \quad \text { and } \\
n_{1} & =-s^{4}(s-1)^{2}\left(u+s^{2}(s-1)\right) / u^{2},
\end{aligned}
$$

we apply Algorithm 5 to derive maps $T$ and $N$ from $k[u]$ into $k(u)$; the elements $n_{i, j}$ computed by Algorithm [5 are given in the table below.

$$
\begin{array}{|c|l|}
n_{0,0} & 1 \\
n_{0,1} & s^{2}(s-1)\left(\left(s^{2}-s-1\right) u+s^{2}(s-1)\right) / u^{2} \\
n_{0,2} & (s-1)^{2} s^{4}\left(2 u^{3}+\left(s^{4}-3 s^{2}+2 s+1\right) u^{2}\right. \\
& \left.\quad+2(s-1)\left(s^{2}-s-1\right) s^{2} u+(s-1)^{2} s^{4}\right) / u^{4} \\
n_{1,1} & -s^{4}(s-1)^{2}\left(u+s^{2}(s-1)\right) / u^{2} \\
n_{1,2} & \left(s^{6}(s-1)^{3}\left(s^{2}-s-1\right) u^{2}+s^{9}(s-1)^{5} u+s^{10}(s-1)^{5}\right) / u^{4} \\
n_{2,2} & s^{8}(s-1)^{4}\left(u+s^{2}(s-1)\right)^{2} / u^{4}
\end{array}
$$

The endomorphism $\eta$ is then explicitly realized by $\eta: P \mapsto \operatorname{Evaluate}(P, T, N)$, using Algorithm [ 


\section{Construction of Curves of Cryptographic Proportions}

The curves presented here not only admit efficiently computable endomorphisms, they also permit random selection of curve parameters in a large family. For example, let $\mathbb{F}_{5^{37}}=\mathbb{F}_{5}[\xi]$ be extension of $\mathbb{F}_{5}$ such that $\xi^{37}+4 \xi^{2}+3 \xi+3=0$, and take

$$
t=3 \xi^{5}+\xi^{4}+3 \xi^{3}+\xi^{2}+2 \xi+3
$$

This gives a curve $X: v^{2}=u\left(u^{2}-1\right)^{2}+t$ in the Artin-Schreier family whose Jacobian has nearly prime group order

$$
\left|\operatorname{Jac}(X)\left(\mathbb{F}_{5}[\xi]\right)\right|=5 \cdot n,
$$

with prime cofactor

$$
n=1058791184067701689674637025340531565456011790341311 .
$$

Such curves are amenable to efficient point counting techniques using MonskyWashnitzer cohomology [79]. If $y$ is a square root of $t$, then $(0, y)$ is a point on $X$; let $P=[(u, v-y)]$ be the corresponding point on $J$. Then $Q=[5](P)$ generates a cyclic group of order $n$, on which $[\eta]$ satisfies

$$
\left(\left[\eta_{5}\right]^{2}+\left[\eta_{5}\right]-1\right)(Q)=[(1)]
$$

and in particular, $\left[\eta_{5}\right](5 P)=[m](5 P)$, where

$$
m=336894053941004885519266617028956898972619907667301
$$

is one of the two roots of $x^{2}+x-1 \bmod n$.

Acknowledgement. The authors thank K. Takashima for providing an advance draft of his article [19], and for references to the work of Hashimoto.

\section{References}

1. D. Cantor, Computing in the Jacobian of a hyperelliptic curve, Math. Comp., 48 (1987), 95-101.

2. M. Ciet, T. Lange, F. Sica, and J.-J. Quisquater, Improved algorithms for efficient arithmetic on elliptic curves using fast endomorphisms, Advances in Cryptology EUROCRYPT 2003, 387-400, LNCS 2656, Springer, Berlin, 2003.

3. I. Duursma and K. Sakurai, Efficient algorithms for the Jacobian variety of hyperelliptic curves $y^{2}=x^{p}-x+1$ over a finite field of odd characteristic $p$. Coding theory, cryptography and related areas (Guanajuato, 1998), 73-89, Springer, Berlin, 2000.

4. J. Ellenberg, Endomorphism algebras of Jacobians. Advances in Mathematics 162 (2001), 243-271.

5. J. W. S. Cassels and E. V. Flynn, Prolegomena to a middlebrow arithmetic of curves of genus 2, London Mathematical Society Lecture Note Series 230, Cambridge University Press, Cambridge, 1996. 
6. R. Gallant, R. Lambert, and S. Vanstone, Faster point multiplication on elliptic curves with efficient endomorphisms, Advances in Cryptology - CRYPTO 2001, 190-200, LNCS 2139, Springer, 2001.

7. P. Gaudry and N. Gürel, Counting points in medium characteristic using Kedlaya's algorithm, Experiment. Math. 12 (2003), no. 4, 395-402.

8. K.-I. Hashimoto, On Brumer's family of RM-curves of genus two. Tohoku Math. J. (2) 52, (2000), no. 4, 475-488.

9. K. Kedlaya, Counting points on hyperelliptic curves using Monsky-Washnitzer cohomology, J. Ramanujan Math. Soc. 16 (2001), no. 4, 323-338.

10. N. Koblitz, CM-curves with good cryptographic properties. Advances in Cryptology - CRYPTO '91, 279-287, LNCS 576, Springer, 1992.

11. T. Lange, Efficient arithmetic on hyperelliptic Koblitz curves, Ph.D. Thesis, 2001.

12. R. Lidl, G. L. Mullen and G. Turnwald, Dickson polynomials, Pitman monographs and surveys in pure and applied mathematics 65, Longman Scientific \& Technical, 1993.

13. A. J. Menezes and S. A. Vanstone, The implementation of elliptic curve cryptosystems, Advances in Cryptology - Auscrypt 1990, 2-13, LNCS 453, Springer-Verlag, 1990.

14. J.-F. Mestre, Familles de courbes hyperelliptiques à multiplications réelles, Arithmetic algebraic geometry (Texel, 1989), 193-208, Progress in Math., 89, Birkhäuser Boston, Boston, MA, 1991.

15. Y.-H. Park, S. Jeong, J. Lim, Speeding up point multiplication on hyperelliptic curves with efficiently-computable endomorphisms, Advances in cryptology - EUROCRYPT 2002 (Amsterdam), 197-208, LNCS 2332, Springer, 2002.

16. B. A. Smith, Explicit endomorphisms and correspondences, Ph.D. Thesis, The University of Sydney, 2005.

17. J. A. Solinas, Efficient arithmetic on Koblitz curves, Des. Codes Cryptogr, 19 (2000), no. 2-3, 195-249.

18. M. Stoll, Two simple 2-dimensional abelian varieties defined over $Q$ with MordellWeil group of rank at least 19, C. R. Acad. Sci. Paris Sér. I Math. 321 (1995), no. $10,1341-1345$.

19. K. Takashima, A new type of fast endomorphisms on Jacobians of hyperelliptic curves and their cryptographic application. IEICE Trans. Fundamentals, E89-A (2006), no. 1, pp. 124-133.

20. W. Tautz, J. Top, A. Verberkmoes, Explicit hyperelliptic curves with real multiplication and permutation polynomials, Canad. J. Math., 43 (1991), no. 5, 1055-1064.

21. A. Weil, On some exponential sums, Proc. Nat. Acad. Sci., 34, (1948), 204-207. 